\section{Are comet spins primordial?}

SiR-Ferrin ${ }^{1}$ contends that several comets, including Halley, rotate at rates determined during their primordial accretion. He bases this on the fact that comets satisfy $L / M \approx M^{2 / 3}$ ( $L$ is spin angular momentum and $M$ is mass), a relation approximately fulfilled by virtually all other solar system members. From this, he infers very low densities $\varrho$ for several comets.

Here, I argue that (1) the $L / M$ relation indicates only that spin periods $T$ of most Solar System members are about the same; (2) deviations from the constantspin rule in other small Solar System bodies can be attributed to current processes; (3) the spin rates of asteroids and comets have been significantly altered by collisions and outgassing, respectively, and are thus not primordial; and (4) the $L /$ $M$ relation, although perhaps useful as a crude guide, is not a reliable indicator of density for a specific object.

For a homogeneous rotating sphere, $L /$ $M=0.97 M^{2 / 3} /\left(T \varrho^{2 / 3}\right)$; a similar expression is known for triaxial ellipsoids ${ }^{1.2}$. Thus, if density differences between different celestial bodies can be ignored, the observation that $L / M \approx M^{\prime 3}$ implies that rotation periods of Solar System members are roughly the same, $\sim 8 \mathrm{~h}$, as originally noted for 70 asteroids and most planets ${ }^{2}$.

Rotation periods of cometary nuclei are poorly known because the classical halo method $^{3}$ and precession model $^{4}$ are of unproved accuracy. Except for Halley's case, the only reliable values come from recent CCD observations of three cometary nucle $i^{5}$ whose rotation periods are 12$22 \mathrm{~h}$, significantly shorter than the $30-50$ h periods Ferrin used. As cometary data are so scant, one might ask whether the spins of other small Solar System objects say anything about their beginnings.

The spins of almost 500 asteroids are now known ${ }^{6}$. All but $1-2 \%$ have periods within a factor of 3 of the mean, $<T>$ $\sim 9-10 \mathrm{~h}$, similar to most planets, a few nontidally despun satellites, and probably not too far from cometary periods. Furthermore, the considerable variations in asteroidal spins with their size $^{6}$ belies Ferrin's claim that celestial rotations are fixed primordially. The data show that: (1) large asteroids (radius $R \geqslant 62.5 \mathrm{~km}$ ) have $\langle T\rangle \approx 7.5 \mathrm{~h}$ and a maxwellian distribution of spin rates, implying that collisions are important; (2) minor planets with $R \approx 40-50 \mathrm{~km}$ spin more slowly $(<T\rangle \approx 11 \mathrm{~h}$ ), a fact generally ascribed to angular momentum drain'; (3) the smallest asteroids $(R<15 \mathrm{~km})$ rotate faster in the mean $(<T>\approx 8.5 \mathrm{~h})$ but also have a wider dispersion, especially showing an excess of slow rotators; they do not obey a maxwellian distribution, perhaps indicating their birth in catastrophic disruption events ${ }^{6}$; (4) spin characteristics also vary with asteroid taxonomy, with M-asteroids, presumed to be metal-rich and relatively dense, rotating fastest ${ }^{6}$.

Small asteroids and comets have had significant angular momentum imparted to them over the aeons. For the former, simple particle-in-a-box calculations ${ }^{8}$ find frequent mutual collisions, both erosive and catastrophic, that re-set rotation rates, expecially those of the tiniest asteroids. In contrast, comets rarely encounter a compatriot in the spacious Oort cloud nor often meet other projectiles during their brief sojourns within the inner Solar System, so collisions do not appreciably alter their angular momentum. Asymmetric outgassing may however induce comets to twirl faster or slower. The same gas jets that cause the non-gravitational accelerations of cometary orbits produce torques capable of modifying spins. Taking the tangential outgassing reaction force to be $10^{-6}$ of solar gravity and, for a lower bound on de-spin time, considering this force to have a moment $\operatorname{arm} R$, spin rates are found to change dramatically over only a few orbits. A similarly swift de-spinning comes from estimating the angular momentum carried away by the sublimated gas. Because expansion velocities in jets are a few hundred $\mathrm{m} \mathrm{s}^{-1}$ whereas typical surface speeds are $\sim 1$ $\mathrm{m} \mathrm{s}^{-1}$, much less than one per cent of the comet's mass, if lost tangentially to the surface, could remove the body's spin angular momentum. Such a mass is expelled in just a few passes by the Sun.

The rough rule that celestial objects whether asteroids, comets or pulsars spin faster when they are denser simply manifests the greater ability of compact objects to hold themselves together as they are spun up. Equatorial surface layers on solid spheres become centrifugally unbound once $T \leqslant 3.3 \quad(\varrho / g$ $\left.\mathrm{cm}^{-3}\right)^{-1 / 2} \mathrm{~h}$ (refs $2,3,5$ ) while debris on the most distant tips of triaxial bodies will be lost at even slower rates ${ }^{2}$. Loosely bound agglomerates (rubble piles), such as sometimes proposed for comets ${ }^{11}$, flow and elongate at spin periods less than $\sim 6(\varrho / g$ $\left.\mathrm{cm}^{-3}\right)^{-1 / 2} \mathrm{~h}$ (ref. 12).

Whether one looks at Solar System rotation data in terms of a constant spinrate law or some universal $L / M$ plot, one should not take the results too literally. While appearing profound, $L / M$ relations, which also seem to apply to other classes of astronomical objects, have been dismissed $^{13}$ because they merely reflect reasonable upper and lower limits on orbital and/or spin rates, much as I maintain above. Furthermore, data that look so remarkable on log-log plots over many orders of magnitude in the mass actually show considerable scatter of individual points. As an extreme example, 288 Glauke, an $18.5-\mathrm{km}$ S-object, takes $1,150 \mathrm{~h}$ to rotate while the comparable asteroid 321 Florentina spins in $2.87 \mathrm{~h}$; are we to conclude that their densities differ by a factor of 8,000 ?

These arguments show that the observed rotations of comets and small asteroids are not primordial. Instead, their spins probably result from a partly delimited evolution. Torques, which act sporadically to spin these bodies up or down, cannot produce very rapid rotations because of the centrifugal breakup limit; the end result is that spins are always found somewhere between zero and this limit. Clearly for asteroids, rotation rates do hint at processes of angular momentum transfer. But too little is now known about cometary rotation for cometary origins or properties to be valuably constrained.

On the other hand, it may be that, as the Duchess in Alice in Wonderland believed, "If everybody minded their own business, the world would go round a great deal faster than it does."

\section{Departments of Astronomy and of Theoretical and Applied Mechanics, Cornell University,}

Ithaca, New York 14853, USA

1. Ferrin, I. Nature 333, 834-835 (1988).

2. Burns, J.A. icarus 25, 545-554 (1975).

3. Whipple, F.L. in Comets (ed. Wilkening, L.L.), 227-250 (Univ. Arizona Press, Tucson, 1982).

4. Sekanina, Z. Ast. J. 95, 1876-1894 (1988).

5. Jewitt, D.C. \& Meech, K.J. Astrophys. J328, 974-986 (1988).

6. Binzel, R.P., Farinella, P., Zappalà, V. \& Cellino, A. in Asteroids // (eds Binzel, R.P., Gehrels, T. \& Matthews, M.S.) (Univ. Arizona Press, Tucson, in the press).

7. Dobrovolskis, A.R. \& Burns, J.A. Icarus 57, 464-476 (1984).

8. Burns, J.A. \& Tedesco, E.F. in Asteroids (ed. Gehrels, T.). 494-527, (Univ. Arizona Press, Tucson, 1979).

9. Marsden, B.G., Sekanina, Z. \& Yeomans, D.K. Astr. J. 78 , 211-225 (1973).

10. Wallis, M.K. in Comets (ed. Wilkening, L.L.), 358-369 (Univ Arizona Press, Tucson, 1982).

11. Weissman, P.R. Nature 320, 242-243 (1986)

12. Weidenschilling. S.J. Icarus 46, 124-126 (1981)

13. Trimble, V. Comments Astrophys. 10, 127-135 (1984)

\section{On Prochlorothrix}

Sir-In his News and Views article', David Penny noted the conflicting results of two comparative biochemical studies of Prochlorothrix and other photosynthetic prokaryotes - a study by Turner et al. ${ }^{2}$ on the $16 \mathrm{~S}$ ribosomal RNA subunit and a study by Morden and Golden ${ }^{3}$ on psbA, a photosystem II-associated protein. Turner et al. place Prochlorothrix close to Synecococcus in a separate line of descent from green chloroplasts, whereas Morden and Golden suggest a closer relationship between green chloroplasts and Prochlorothrix. Although these results are inconsistent, it should be noted that both studies $^{2.3}$ are a preliminary part of the effort to place the Prochlorophytes on the basis of the limited amount of available 